\title{
Absence of Pharmacokinetic Interactions between the Bruton's Tyrosine Kinase Inhibitor Fenebrutinib and Methotrexate ${ }^{[\rrbracket}$
}

\author{
Nicholas Steven Jones, Helen Winter, Tamiko R. Katsumoto, Marilyn Florero, Elaine Murray, \\ Helen Walker, Nand Singh, and Leslie W. Chinn \\ Clinical Science (N.J., T.K., M.F.), Clinical Pharmacology (H.Wi., L.C.), and Safety Science (E.M.), Genentech, Inc., South San \\ Francisco, California; and Quotient Sciences, Ruddington, Nottingham, United Kingdom (H.Wa., N.S.)
}

Received February 11, 2019; accepted July 26, 2019

\section{ABSTRACT}

Fenebrutinib (GDC-0853) is an orally administered small molecule inhibitor of Bruton's tyrosine kinase being investigated for treatment of rheumatoid arthritis in patients with inadequate responses to methotrexate (MTX). This study interrogated the potential for pharmacokinetic drug interactions between fenebrutinib and MTX. Eighteen healthy male subjects were enrolled in the study. They received a single oral dose of MTX $(7.5 \mathrm{mg})$ on day 1 followed by a 13-day washout period. Subsequently, on days 15-20 the participants received $200 \mathrm{mg}$ of fenebrutinib twice daily. On day 21 , they received a $7.5 \mathrm{mg}$ dose of MTX and a $200 \mathrm{mg}$ dose of fenebrutinib under fasting conditions. The geometric mean ratios of MTX area under the plasma concentration-time curve (AUC) and $C_{\max }$ on day 21 relative to day $1(90 \%$ confidence interval $[\mathrm{Cl}])$ were $0.96(0.88-1.04)$ and $1.05(0.94-1.18)$, respectively. The geometric mean ratios of fenebrutinib $A \cup C$ and $C_{\text {max }}$ for day 21 relative to day $20(90 \% \mathrm{Cl})$ were $1.03(0.95-1.11)$ and 1.02 (0.90-1.15), respectively. The combination treatment was well tolerated, with an adverse event profile similar to that reported in other MTX trials. These results indicate that there is no clinically significant pharmacokinetic interaction between fenebrutinib and MTX.

\section{Introduction}

Rheumatoid arthritis (RA) is a chronic autoimmune disorder associated with functional decline, pain, and a reduced quality of life. Between $0.5 \%$ and $1 \%$ of the world's population is affected, and the economic burden of RA is high (Uhlig et al., 2014). RA is characterized by progressive synovitis, systemic inflammation, and the production of characteristic autoantibodies (Lundkvist et al., 2008), which ultimately results in irreversible damage of joint cartilage and bone. Although there are several medications available to treat RA, there is unmet need for safer and more efficacious therapies, ideally leading to full remission of disease (Kjeken et al., 2006; Montag et al., 2011). In response to this need, potential therapies are being evaluated against new molecular targets, including protein kinase inhibitors that interfere with intracellular signaling and the downstream regulation and proliferation of immune cells.

One molecular target of interest is Bruton's tyrosine kinase (BTK), a cytoplasmic protein tyrosine kinase primarily expressed in hematopoietic cells, including macrophages. BTK is a key signaling kinase during B cell-antigen

This work was funded by Genentech, Inc.

https://doi.org/10.1124/jpet.119.257089.

S This article has supplemental material available at jpet.aspetjournals.org. receptor $(\mathrm{BCR})$ activation, and it plays a role in Fc receptor signaling. Inhibition of BTK can result in the prevention of BCR-dependent cell proliferation as well as reduced production of inflammatory cytokines via these two mechanisms (Kelly and Genovese, 2013). Given that BTK is involved in multiple signaling pathways downstream of the BCR and FcR, it represents an attractive therapeutic target for RA and other inflammatory disorders including systemic lupus erythematosus and chronic spontaneous urticaria

GDC-0853 (fenebrutinib) is a highly selective, reversible inhibitor of BTK and is being developed by Genentech as an orally administered treatment of patients with RA, systemic lupus erythematosus, and chronic spontaneous urticaria. Unlike other first-generation BTK inhibitors that act upon several different tyrosine kinases, fenebrutinib binds noncovalently to BTK in an orientation that enables greater selectivity (Crawford et al., 2018). The in vitro cellular potency of fenebrutinib was determined to be $11 \mathrm{nM}$ for the inhibition of BTK autophosphorylation in human whole blood obtained from healthy subjects. Additionally, target engagement has been demonstrated in a multiple ascending dose study in healthy subjects, where a maximal inhibition above $90 \%$ was seen in BTK autophosphorylation and basophil assays (Herman et al., 2018).

ABBREVIATIONS: AE, adverse event; AUC, area under the plasma concentration-time curve; BCR, B cell-antigen receptor; BCRP, breast cancer resistance protein; BTK, Bruton's tyrosine kinase; $\mathrm{Cl}$, confidence interval; DDI, drug-drug interaction; DMARD, disease-modifying antirheumatic drug; GDC-0853, fenebrutinib; LLOQ, lower limit of quantification; MRP, multidrug resistance protein; MTX, methotrexate; NSAID, nonsteroidal anti-inflammatory drug; OAT, organic anion transporter; PK, pharmacokinetics; RA, rheumatoid arthritis; ss, steady state; TEAE, treatment-emergent adverse event. 
As there is currently no cure for RA, treatments focus on providing symptomatic relief of pain and stiffness, thus improving patients' ability to move and their quality of life. Medications known as disease-modifying antirheumatic drugs (DMARD) combat the inflammation of RA. Methotrexate (MTX), an immunosuppressant, is widely regarded as the preferred first-line conventional DMARD for most patients with RA. One of the targets of MTX associated with efficacy in RA is hypothesized to be 5-aminoimidazole-4-carboxamide ribonucleotide transformylase; inhibition of this enzyme and accumulation of substrates are thought to reduce lymphocyte proliferation (Morgan et al., 2004). Low-dose weekly MTX is used in approximately $80 \%$ of patients with RA; therefore, it is important to understand the potential for pharmacokinetic (PK) drug interactions between MTX and candidate compounds being developed for the treatment of RA (Kim et al., 2016).

MTX is a folic acid derivative administered orally or by subcutaneous or intramuscular injection. After oral administration, MTX is rapidly absorbed, with an elimination halflife of approximately 5-8 hours. Methotrexate is mainly excreted unchanged by the kidney and undergoes both secretion and reabsorption within the renal tubules (Bannwarth et al., 1996), rendering it susceptible to alterations in PK through inhibition of transporter proteins involved in these processes. Clinically relevant effects on MTX PK have been reported via the breast cancer resistance protein (BCRP), organic anion transporter 3 (OAT3), and multidrug resistance protein 4 (MRP4) transporters (Ivanyuk et al., 2017). In vitro transporter assay results suggest that fenebrutinib may inhibit BCRP (Jones et al., 2019, https://www.ncbi.nlm. nih.gov/pubmed/?term=31376152). The $\mathrm{IC}_{50}$ value for this interaction is substantially higher than the concentrations that would be anticipated after fenebrutinib administration at doses currently under consideration; however, out of an abundance of caution, the potential effect of fenebrutinib on MTX was assessed before initiation of a phase 2 study in patients with RA on a background MTX regimen.

To evaluate the potential for PK interactions between MTX and fenebrutinib, a phase 1 study was conducted in which single low doses of MTX were administered in the presence or absence of steady-state fenebrutinib. The objectives of this study were to evaluate the potential for drugdrug interactions (DDI) and to characterize the PK, tolerability, and safety of fenebrutinib and MTX in healthy subjects to assess the feasibility of concurrent administration of fenebrutinib and MTX.

\section{Methods}

Study Design. This investigation was a phase 1, single-center, fixed-sequence study with three treatment periods: MTX administered alone, fenebrutinib administered alone, and MTX and fenebrutinib administered in combination. Fenebrutinib tablets were supplied by Quotient Clinical as 50-mg tablets packaged as per product label. Methotrexate (PL 14510/0032; EBEWE Pharma, Unterach am Attersee, Austria) was procured by Quotient Clinical as UK licensed products.

The purposes were to characterize the following: 1) the effect of simultaneous administration of a single dose of MTX on the steadystate PK of fenebrutinib and 2) the effect of dosing fenebrutinib on the single-dose PK of MTX in healthy subjects. The study population consisted of healthy male subjects between the ages of
18 and 55 years, with normal renal function and no laboratory or clinical history of cytopenias or liver enzyme abnormalities. A single dose of $7.5 \mathrm{mg}$ of MTX was administered under fasting conditions on day 1 followed by a 13-day washout period (see Supplemental Fig. 1). Subsequently, $200 \mathrm{mg}$ of fenebrutinib was administered twice daily under fasting conditions on days 15-20, which was considered sufficient to reach steady state. On day 21 , a single dose of $200 \mathrm{mg}$ of fenebrutinib was coadministered with $7.5 \mathrm{mg}$ of MTX under fasting conditions. There was a 20-day washout period between the MTX dose administrations (day 1 and day 21), based on the half-life of MTX which undergoes $>99 \%$ elimination from systemic circulation after 13 days. Folic acid $(5 \mathrm{mg})$ was administered orally approximately 24 hours after each dose of MTX to mitigate the development of potential side effects related to MTX. The MTX dose of $7.5 \mathrm{mg}$ was selected to give a clinically relevant exposure while minimizing the potential of MTX-associated adverse events (AEs). Additionally, this MTX dose has been administered to healthy subjects previously (Namour et al., 2012).

This study was conducted at Quotient Sciences (Nottingham, United Kingdom) according to the International Council for Harmonization (Kim et al., 2016) E6 Guideline for Good Clinical Practice (GCP), the E.U. Clinical Trial Directive (2001/20/EC), and applicable local, state, and federal laws. This study was conducted in full conformance with the principles of the Declaration of Helsinki or the laws and regulations of the country in which the research was conducted; whichever afforded the greater protection to the individual. The study complied with the requirements of the ICH E2A guideline (Clinical Safety Data Management: Definitions and Standards for Expedited Reporting).

Sample Size Calculation. A sample size of at least 15 subjects was required such that the probability that the $90 \%$ confidence interval (CI) for the geometric mean exposure ratios was contained within $80 \%$ and $125 \%$ for MTX and within $50 \%$ and $200 \%$ for fenebrutinib was at least 0.8 . This sample size calculation assumes a within-subject coefficient of variation of $63 \%$ for fenebrutinib and a within-subject coefficient of variation of $28 \%$ for MTX. To allow for possible dropouts, at least 17 subjects were planned to be enrolled.

Analytical Methods. Blood samples for the measurement of MTX were obtained up to 24 hours after the administration of MTX on days 1 and 21. Of note, MTX metabolites have not been conclusively associated with activity (safety or efficacy), so quantification of MTX metabolites was not performed in this study. Blood samples for the measurement of fenebrutinib were obtained before the morning dose of fenebrutinib on days 17,18 , and 19 , and for up to 24 hours after the morning dose of fenebrutinib on days 20 and 21.

Plasma concentrations of fenebrutinib and MTX were determined using validated bioanalytical methods at Covance Laboratories (Madison, WI). The concentrations of fenebrutinib and MTX in human plasma containing $\mathrm{K}_{2}$ EDTA as an anticoagulant were measured using supported-liquid extraction followed by analysis with high performance liquid chromatography followed by tandem mass spectrometric detection (LC-MS/MS). The lower limit of quantification (LLOQ) was $0.5 \mathrm{ng} / \mathrm{ml}$ for fenebrutinib and $1 \mathrm{ng} / \mathrm{ml}$ for MTX. Analysis runs were determined to have a high degree of reproducibility $(97.9 \%$ of repeated results were within $20 \%$ of the original results) and a low degree of interrun carryover $(<5 \%)$, which was considered acceptable for pharmacokinetic quantitation.

Determination of Pharmacokinetic Parameters. Plasma concentrations of fenebrutinib and MTX and sampling times after dosing were used to estimate PK parameters of both fenebrutinib and MTX using Phoenix WinNonlin PK software (v6.3; Certara USA, St. Louis, MO). Profiles of mean plasma concentrations against sampling times after dosing were generated. The estimated PK parameters included the area under the plasma concentration-time curve (AUC), the maximal concentration observed in plasma $\left(C_{\max }\right)$, and the time after dosing at which $C_{\max }$ was apparent $\left(t_{\max }\right)$ for fenebrutinib and MTX. Summary statistics (i.e., mean, median, CV\%, $n$, minimum, maximum, and geometric mean) were calculated for fenebrutinib 


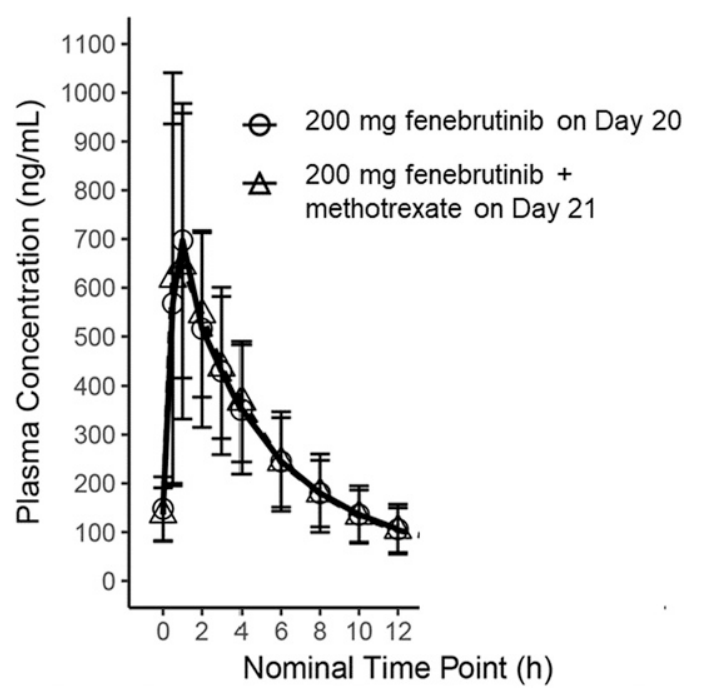

Fig. 1. Mean ( \pm S.D.) plasma GDC-0853 (fenebrutinib) concentrationtime profiles over 12 hours in healthy subjects $(n=13)$ on day 20 and day 21 after multiple twice-daily doses of $200 \mathrm{mg}$ of fenebrutinib under fasting conditions on days $15-20$, and a single final dose of $200 \mathrm{mg}$ of fenebrutinib coadministered with $7.5 \mathrm{mg}$ of MTX (methotrexate) under fasting conditions on day 21 .

and MTX PK parameters as applicable. Concentrations below the LLOQ were set to 0 for the calculation of summary statistics except for calculation of geometric summary statistics, where values were set to $0.5 \times$ LLOQ. Mean concentration-time curves by treatment/analyte were generated.

Safety Assessment. The incidence of AEs was monitored throughout the study. An AE was defined as any unfavorable or untoward medical occurrence in a clinical investigational subject administered a pharmaceutical product, regardless of causal attribution. AEs were assessed based on severity, duration, and relationship to the study drug.

Statistical Analysis. Formal statistical analysis was performed on the following plasma $\mathrm{PK}$ parameters: $\mathrm{AUC}_{0-12, \mathrm{ss}}$ and $C_{\text {max,ss }}$ for fenebrutinib, and $\mathrm{AUC}_{0-24}$ and $C_{\max }$ for MTX. The PK parameters were logarithmically transformed and were subsequently analyzed using ANOVA modeling techniques. The model included terms for treatment as a fixed effect and subject as a random effect. The following pairwise treatment comparisons were analyzed: 1) comparison of fenebrutinib at steady state coadministered with MTX (day 21) versus fenebrutinib administered alone at steady state (day 20) and 2) comparison of MTX coadministered with fenebrutinib (day 21) versus MTX administered alone (day 1). Adjusted geometric mean ratios and $90 \%$ CIs for the adjusted geometric mean ratios for the pairwise treatment comparisons were calculated. No adjustment for multiple comparisons was performed.

\section{Results}

Patient Demographics and Baseline Characteristics. A total of 18 healthy male subjects with a mean age of 32 years (range: 20-55 years) participated, and 12 of them successfully completed the study. All 18 subjects received at least 1 dose of MTX or fenebrutinib and were therefore included in the safety population. Six subjects discontinued the study: two withdrew consent, and four discontinued due to mild treatment-emergent adverse events (TEAEs). Thirteen subjects completed all treatments (one subject completed all doses but was lost to follow up before the final assessment and was therefore considered a study discontinuation), and they contributed at least three quantifiable postdose plasma concentrations on days 1,20 , and 21 . They constituted the PK population for assessment of a DDI. Fifteen subjects were white Caucasians, two subjects were of African ethnic origin, and one subject was Asian (Bangladeshi). Baseline inflammatory markers (erythrocyte sedimentation rate and C-reactive protein) were within the normal range for all subjects, and creatinine clearance results were recorded for all subjects and were $>90 \mathrm{ml} / \mathrm{min}$. The baseline demographics are described in the supplemental materials (Supplemental Table 1).

Pharmacokinetics of Fenebrutinib. Overall, a single dose of $7.5 \mathrm{mg}$ MTX produced no significant effect on the steady-state plasma concentrations of fenebrutinib; the mean fenebrutinib concentration-time profiles in the presence and absence of MTX are presented on a linear/linear scale in Fig. 1.

The key PK parameters for fenebrutinib after multiple oral doses of $200 \mathrm{mg}$ of fenebrutinib and coadministration with 7.5 mg of MTX in the fasting state are shown in Table 1. Observation of mean predose fenebrutinib concentrations indicated that a steady state was reached by day 19. At steady state, the fenebrutinib $t_{\max }$ ranged from 0.50 to 2 hours, with a median value of 1 hour. The geometric mean (CV\%) steady state fenebrutinib $C_{\max }$ and $\mathrm{AUC}_{0-12}$ values were $659 \mathrm{ng} / \mathrm{ml}$ (54.1\%) and $3270 \mathrm{ng} . \mathrm{h} / \mathrm{ml}(45.2 \%)$, respectively. After $C_{\max }$, plasma fenebrutinib concentrations declined with a geometric mean terminal half-life of 4.63 hours (16.0\%). On day 21, after coadministration with $7.5 \mathrm{mg}$ MTX, the fenebrutinib $t_{\max }$ ranged from 0.50 to 2 hours, with a median value of 0.50 hours. The geometric mean (CV\%) fenebrutinib $C_{\max }$ and $\mathrm{AUC}_{0-12}$ values at steady state were $673 \mathrm{ng} / \mathrm{ml}(51.4 \%)$ and $3360 \mathrm{ng} . \mathrm{h} / \mathrm{ml}$ $(39.2 \%)$, respectively, and the geometric mean (CV\%) terminal half-life was 6.87 hours $(28.5 \%)$. The geometric mean ratios $(90 \% \mathrm{CI})$ for fenebrutinib $C_{\text {max,ss }}$ and $\mathrm{AUC}_{0-12, \mathrm{ss}}$ were 1.02 (0.90-1.15) and $1.03(0.95-1.11)$, respectively, in the presence of MTX compared with administration alone.

Pharmacokinetics of Methotrexate. The mean plasma MTX concentration-time profiles after oral administration of a single dose of $7.5 \mathrm{mg}$ of MTX on day 1 and with

\section{TABLE 1}

Steady-state GDC-0853 (fenebrutinib) pharmacokinetic parameters (geometric mean, CV\%) for BID fenebrutinib administered alone on day 20 , and in combination with a single dose of $7.5 \mathrm{mg}$ of methotrexate (MTX) on day 21

\begin{tabular}{lcc}
\hline \multirow{2}{*}{ PK Parameter } & \multicolumn{2}{c}{ 200-mg Fenebrutinib } \\
\cline { 2 - 3 } & $\begin{array}{c}\text { Day } 20, \text { Fasting } \\
(n=13)^{a}\end{array}$ & $\begin{array}{c}+7.5 \text { mg MTX } \\
\text { Day 21, Fasting }(n=13)^{b}\end{array}$ \\
\hline$t_{\text {max,ss }}(\mathrm{h})^{c}$ & $1.00(0.50-2.00)$ & $0.50(0.50-2.00)$ \\
$C_{\text {max,ss }}(\mathrm{ng} / \mathrm{ml})$ & $659(54.1)$ & $673(51.4)$ \\
$\mathrm{AUC}_{0-12, \mathrm{ss}}(\mathrm{ng} \cdot \mathrm{h} / \mathrm{ml})$ & $3270(45.2)$ & $3360(39.2)$ \\
$t_{1 / 2, \mathrm{ss}}(\mathrm{h})$ & $4.63(16.0)$ & $6.87(28.5)$ \\
Geometric mean ratio of & $\mathrm{NA}$ & $1.03(0.95-1.11)$ \\
AUC $_{0-12, \mathrm{ss}}(90 \% \mathrm{CI})$ & $\mathrm{NA}$ & $1.02(0.90-1.15)$ \\
Geometric mean ratio of & & \\
$C_{\text {max,ss }}(90 \%$ CI \%) & & \\
\hline
\end{tabular}

AUC, area under the plasma concentration-time curve; BID, twice daily; CI, confidence interval; NA, not applicable; PK, pharmacokinetic.

${ }^{a}$ Single dose of $200 \mathrm{mg}$ of fenebrutinib BID on days $15-20$ under fasting conditions (an overnight fast for the morning dose and a 2-hour fast for the evening dose).

${ }^{b}$ Single dose of $200 \mathrm{mg}$ of fenebrutinib (along with $7.5 \mathrm{mg}$ of MTX) on day 21 under fasting conditions.

${ }^{c}$ Median (range) 


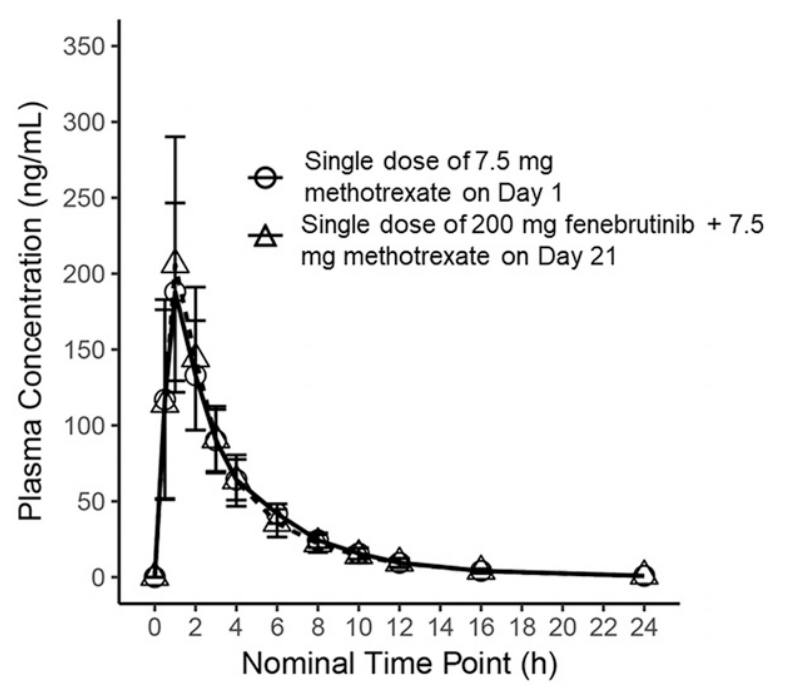

Fig. 2. Mean ( \pm S.D.) plasma methotrexate (MTX) concentration-time profile in healthy subjects $(n=13)$ after a single dose of $7.5 \mathrm{mg}$ of MTX under fasting conditions on day 1 , and a single dose of $7.5 \mathrm{mg}$ of MTX coadministered with $200 \mathrm{mg}$ of GDC-0853 (fenebrutinib) under fasting conditions on day 21 .

coadministration of $200 \mathrm{mg}$ of fenebrutinib on day 21 were superimposable (Fig. 2).

The key PK parameters for MTX after a single oral dose of $7.5 \mathrm{mg}$ of MTX in the presence and absence of steady-state concentrations of fenebrutinib are shown in Table 2. After a single oral dose of $7.5 \mathrm{mg}$ of MTX, MTX plasma concentrations were measured at the first postdose time point ( 0.50 hours) and remained quantifiable until at least 16 hours after the dose in all subjects. The MTX $t_{\max }$ occurred between 0.50 and 2 hours with a median of 1 hour after the dose. The geometric mean $(\mathrm{CV} \%)$ MTX $C_{\max }$ and $\mathrm{AUC}_{0-24}$ were $191 \mathrm{ng} / \mathrm{ml}$ (28.2\%) and $730 \mathrm{ng} . \mathrm{h} / \mathrm{ml}$ (15.2\%), respectively. After $C_{\max }$, MTX plasma concentrations declined in a monophasic or a biphasic manner, with a geometric mean (CV\%) terminal half-life of 3.42 hours $(20.4 \%)$, consistent with the expected PK (Bannwarth et al., 1996).

After coadministration with $200 \mathrm{mg}$ of fenebrutinib on day 21 , the MTX $t_{\max }$ occurred between 1.00 and 2.00 hours, with a median of 1.00 hour after the dose. The geometric mean
$(\mathrm{CV} \%)$ MTX $C_{\max }$ and $\mathrm{AUC}_{0-24}$ were $220 \mathrm{ng} / \mathrm{ml}(27.8 \%)$ and 724 ng.h/ml (23.6\%), respectively. After reaching $C_{\max }$, the plasma MTX concentrations declined in a monophasic or a biphasic manner, with a geometric mean (CV\%) terminal half-life of 3.57 hours (14.9\%).

The geometric mean ratios $(90 \% \mathrm{CI})$ for MTX $C_{\max }$ and $\mathrm{AUC}_{0-24}$ were $1.05(0.94-1.18)$ and 0.96 (0.88-1.04), respectively, in the presence of fenebrutinib compared with administration of MTX alone.

There were no statistically significant differences in the total or peak exposures of fenebrutinib or MTX after coadministration compared with administration of fenebrutinib or MTX alone.

Safety and Tolerability. This study assessed the safety and tolerability of multiple 200-mg oral doses of fenebrutinib and a single dose of MTX administered in the presence of steady state fenebrutinib in healthy male subjects. fenebrutinib was well-tolerated when given as 200-mg oral doses twice daily in the fasting state for 6 days and with a single 7.5-mg oral dose of MTX. The majority of AEs were mild and were not considered related to fenebrutinib. Two moderate severity unrelated TEAEs-a hand fracture and ligament sprainwere reported in one subject. There were no serious or severe AEs and no deaths.

Twelve out of 18 subjects $(66.7 \%)$ reported 26 AEs (tabulated in Supplemental Table 2). The AEs were reported most commonly during the period of twice daily dosing with fenebrutinib ( 7 of $16,43 \%$ ) with only one $\mathrm{AE}$ (rash) reported after the addition of the single dose of MTX. Overall, the most common events were reported in the skin and subcutaneous disorders system organ class ( 6 of $26,33.3 \%$ ) and consisted of mild AEs of rash (rash, two subjects; rash maculopapular, one subject; and rash pruritic, one subject), generalized pruritus (one subject), and petechiae and papules (one subject). The AE terms were reported once only, with the exception of rash and headache in two subjects each.

Four subjects $(22.2 \%)$ experienced six mild TEAEs that resulted in study drug withdrawal and the subjects' discontinuation from the study. Three of these subjects withdrew during the period of fenebrutinib dosing, one after the initial MTX dosing, and none in the period after dosing with MTX in the setting of steady state fenebrutinib. These TEAEs leading to withdrawal were generalized pruritus, folliculitis,

TABLE 2

Single-dose methotrexate (MTX) pharmacokinetic parameters (geometric mean, CV\%) after oral administration of $7.5 \mathrm{mg}$ MTX on day 1 and in combination with steady-state administration of GDC-0853 (fenebrutinib, $200 \mathrm{mg}$ twice daily) on day 21

\begin{tabular}{lcc}
\hline & & $7.5 \mathrm{mg}$ MTX \\
\cline { 2 - 3 } PK Parameter & Day 1, Fasting $(n=13)^{a}$ & +200 mg Fenebrutinib Day 21, Fasting $(n=13)^{b}$ \\
\hline$t_{\max }(\mathrm{h})^{c}$ & $1.00(0.50-2.00)$ & $1.00(1.00-2.00)$ \\
$C_{\max }(\mathrm{ng} / \mathrm{ml})$ & $191(28.2)$ & $220(27.8)$ \\
$\mathrm{AUC}_{0-t}(\mathrm{ng} \cdot \mathrm{h} / \mathrm{ml})$ & $725(15.8)$ & $718(24.2)$ \\
$\mathrm{AUC}_{0-24}(\mathrm{ng} \cdot \mathrm{h} / \mathrm{ml})$ & $730(15.2)$ & $724(23.6)$ \\
$\mathrm{AUC}$ & $729(23.8)$ \\
$t_{1 / 2}(\mathrm{~h})$ & $736(15.5)$ & $3.57(14.9)$ \\
Geometric mean ratio of $\mathrm{AUC}_{0-24}(90 \% \mathrm{CI})$ & $3.42(20.4)$ & $0.96(0.88-1.04)$ \\
Geometric mean ratio of $C_{\text {max }}(90 \% \mathrm{CI})$ & $\mathrm{NA}$ & $1.05(0.94-1.18)$ \\
\hline
\end{tabular}

AUC, area under the plasma concentration-time curve; BID, twice daily; CI, confidence interval; NA, not applicable; PK, pharmacokinetic. ${ }^{a}$ Single dose of $7.5 \mathrm{mg}$ of MTX administered as $3 \times 2.5 \mathrm{mg}$ tablets under fasting conditions.

${ }^{b}$ Single dose of $7.5 \mathrm{mg}$ of MTX (administered as $3 \times 2.5 \mathrm{mg}$ tablets) coadministered with fenebrutinib under fasting conditions.

${ }^{c}$ Median (range) 
petechiae, pyrexia, influenza-like symptoms, and diarrhea of which only petechiae was considered related to study drug.

All AEs had resolved by the end of the study. There were no clinically significant changes in laboratory parameters, vital signs, ECGs, or physical examination findings recorded during the study.

\section{Discussion}

This phase 1, single-center, fixed-sequence study with two treatments (MTX and fenebrutinib, alone and in combination) in healthy subjects demonstrated that fenebrutinib was well tolerated when a single dose of MTX was coadministered. The effects on the steady state PK of $200 \mathrm{mg}$ of fenebrutinib were minimal when dosed with $7.5 \mathrm{mg}$ of MTX on day 21 . Fenebrutinib $t_{\max }, C_{\max }$, and AUC were comparable with and without MTX, and formal statistical analysis confirmed no significant difference in exposure parameters. The geometric mean half-life for fenebrutinib when coadministered with MTX was 1.5-fold longer than that for a fenebrutinib tablet administered without MTX (6.87 and 4.63 hours, respectively). However, this was likely due to the longer sampling period on day 21 resulting in better definition of the terminal phase and half-life.

MTX is a cornerstone of therapy in the treatment of RA. Hence, determining whether concomitant administration of fenebrutinib affects the PK of MTX was a crucial step before conducting a clinical study in RA patients. Minimal effect was seen on the PK of MTX when coadministered with fenebrutinib at steady state on day 21 , and formal statistical analysis confirmed no significant differences in exposure parameters. MTX $t_{\max }, C_{\max }$, and AUC were comparable in the presence and absence of fenebrutinib. Therefore, fenebrutinib did not appear to significantly impact the PK of MTX.

The safety profile observed during coadministration of fenebrutinib with MTX was typical of that expected from similar MTX DDI studies (Namour et al., 2012; Mohamed et al., 2016; Lee et al., 2017) and from MTX monotherapy (Salliot and van der Heijde, 2009). fenebrutinib was well tolerated when administered as multiple 200 -mg oral doses of the tablet formulation twice daily and with a single 7.5mg oral dose of MTX. Almost all the TEAEs reported were mild, with no serious or severe AEs and no clinically significant changes in laboratory or other parameters recorded during the study. These results combined with the lack of significant changes in PK parameters suggest that fenebrutinib can be administered to RA patients receiving MTX as a background therapy without restrictions or dosage adjustments.

There have been several reports of interactions of small molecule drugs with low-dose MTX, although the clinical relevance of such interactions in the overall RA patient population is unknown (Hall et al., 2017). Typically, MTX is the victim of these interactions, and there is no compelling evidence of cytochrome P450 inhibition by MTX (Baumhäkel et al., 2001). Nonselective nonsteroidal anti-inflammatory drugs (NSAIDs) (nonsteroidal anti-inflammatory drugs), particularly salicylates, have been reported to interact with MTX when administered concomitantly, resulting in increased plasma concentrations and decreased renal clearance of MTX. Some drugs (NSAIDs) elevate and prolong serum MTX levels by reducing tubular secretion via OAT3 and MRP4 (Bannwarth et al., 1996; Ivanyuk et al., 2017). Other drugs (e.g., salicylates, phenylbutazone, phenytoin, and sulfonamides) are reported to increase MTX toxicity by displacing albumin-bound MTX, but protein binding changes in general are rarely considered clinically significant (Benet and Hoener, 2002).

MTX is eliminated primarily via renal excretion. This occurs predominantly via glomerular filtration and with an additional active secretory process via OATs (Songsiridej and Furst, 1990). It is thought that the BCRP transporter is responsible for MTX secretion in the kidneys during the process of renal excretion. A study by Breedveld et al. (2004) suggested that competition for BCRP may explain the DDI between MTX and benzimidazoles (e.g., proton pump inhibitors). In the context of planned evaluation of fenebrutinib in patients with RA for whom MTX is commonly prescribed, and given several reports about the interaction between small molecules and MTX via renal transporters, this study was conducted to assess whether there would be a PK interaction between fenebrutinib and MTX at clinically relevant doses. As fenebrutinib could potentially be used in combination with MTX, it was important to understand the potential for DDIs between these two compounds.

In conclusion, this study demonstrated that concomitant administration of fenebrutinib and MTX resulted in no statistically significant differences in the exposure of fenebrutinib after administration of a single dose of MTX, or in the exposure of MTX after administration of multiple doses of fenebrutinib. These data indicate that fenebrutinib does not substantially affect the systemic exposure of MTX, and that MTX does not significantly change the systemic exposure of fenebrutinib. A favorable tolerability and safety profile also supports concomitant use of MTX and fenebrutinib in patients with autoimmune diseases.

\section{Acknowledgments}

The authors acknowledge Mindy Sivasubramanian, Mario Aceves, and Robin Noguchi (Genentech, Inc.) and Sue Melbourne (Quotient Clinical) for their support of study conduct. The authors acknowledge Gaohong She (Genentech, Inc.) for support of data analysis.

\section{Authorship Contributions}

Participated in research design: Winter, Katsumoto, Florero, Murray, Chinn.

Conducted experiments: Walker, Singh.

Performed data analysis: Jones, Winter, Katsumoto, Florero, Murray, Walker, Singh, Chinn.

Wrote or contributed to the writing of the manuscript: Jones, Winter, Katsumoto, Florero, Murray, Walker, Singh, Chinn.

\section{References}

Bannwarth B, Péhourcq F, Schaeverbeke T, and Dehais J (1996) Clinical pharmacokinetics of low-dose pulse methotrexate in rheumatoid arthritis. Clin Pharmacokinet 30:194-210.

Baumhäkel M, Kasel D, Rao-Schymanski RA, Böcker R, Beckurts KT, Zaigler M, Barthold D, and Fuhr U (2001) Screening for inhibitory effects of antineoplastic agents on CYP3A4 in human liver microsomes. Int J Clin Pharmacol Ther 39: $517-528$.

Benet LZ and Hoener BA (2002) Changes in plasma protein binding have little clinical relevance. Clin Pharmacol Ther 71:115-121.

Breedveld P, Zelcer N, Pluim D, Sönmezer O, Tibben MM, Beijnen JH, Schinkel AH, van Tellingen O, Borst P, and Schellens JH (2004) Mechanism of the pharmacokinetic interaction between methotrexate and benzimidazoles: potential role for breast cancer resistance protein in clinical drug-drug interactions. Cancer Res 64: 5804-5811.

Crawford JJ, Johnson AR, Misner DL, Belmont LD, Castanedo G, Choy R, Coraggio M, Dong L, Eigenbrot C, Erickson R, et al. (2018) Discovery of GDC-0853: a potent, 
selective, and noncovalent Bruton's tyrosine kinase inhibitor in early clinical development. J Med Chem 61:2227-2245.

Hall JJ, Bolina M, Chatterley T, and Jamali F (2017) Interaction between low-dose methotrexate and nonsteroidal anti-inflammatory drugs, penicillins, and proton pump inhibitors. Ann Pharmacother 51:163-178.

Herman AE, Chinn LW, Kotwal SG, Murray ER, Zhao R, Florero M, Lin A, Moein A, Wang R, Bremer M, et al. (2018) Safety, pharmacokinetics, and pharmacodynamics in healthy volunteers treated with GDC-0853, a selective reversible Bruton's tyrosine kinase inhibitor. Clin Pharmacol Ther 103:1020-1028.

Ivanyuk A, Livio F, Biollaz J, and Buclin T (2017) Renal drug transporters and drug interactions. Clin Pharmacokinet 56:825-892.

Kelly V and Genovese M (2013) Novel small molecule therapeutics in rheumatoid arthritis. Rheumatology (Oxford) 52:1155-1162.

Kim G, Barner JC, Rascati K, and Richards K (2016) Examining time to initiation of biologic disease-modifying antirheumatic drugs and medication adherence and persistence among Texas medicaid recipients with rheumatoid arthritis. Clin Ther 38:646-654.

Kjeken I, Dagfinrud H, Mowinckel P, Uhlig T, Kvien TK, and Finset A (2006) Rheumatology care: involvement in medical decisions, received information, satisfaction with care, and unmet health care needs in patients with rheumatoid arthritis and ankylosing spondylitis. Arthritis Rheum 55:394-401.

Lee SK, Xing J, Catlett IM, Adamczyk R, Griffies A, Liu A, Murthy B, and Nowak M (2017) Safety, pharmacokinetics, and pharmacodynamics of BMS-986142, a novel reversible BTK inhibitor, in healthy participants. Eur J Clin Pharmacol 73:689-698.

Lundkvist J, Kastäng F, and Kobelt G (2008) The burden of rheumatoid arthritis and access to treatment: health burden and costs. Eur $J$ Health Econ 8 (Suppl 2): S49-S60.
Mohamed MF, Camp HS, Jiang P, Padley RJ, Asatryan A, and Othman AA (2016) Pharmacokinetics, safety and tolerability of ABT-494, a novel selective JAK 1 inhibitor, in healthy volunteers and subjects with rheumatoid arthritis. Clin Pharmacokinet 55:1547-1558.

Montag K, Gingold M, Boers A, and Littlejohn G (2011) Disease-modifying antirheumatic drug usage, prescribing patterns and disease activity in rheumatoid arthritis patients in community-based practice. Intern Med $J$ 41:450-455.

Morgan SL, Oster RA, Lee JY, Alarcón GS, and Baggott JE (2004) The effect of folic acid and folinic acid supplements on purine metabolism in methotrexate-treated rheumatoid arthritis. Arthritis Rheum 50:3104-3111.

Namour F, Vanhoutte FP, Beetens J, Blockhuys S, De Weer M, and Wigerinck P (2012) Pharmacokinetics, safety, and tolerability of GLPG0259, a mitogenactivated protein kinase-activated protein kinase 5 (MAPKAPK5) inhibitor, given as single and multiple doses to healthy male subjects. Drugs $R D$ 12:141-163.

Salliot C and van der Heijde D (2009) Long-term safety of methotrexate monotherapy in patients with rheumatoid arthritis: a systematic literature research. Ann Rheum Dis 68:1100-1104.

Songsiridej N and Furst DE (1990) Methotrexate--the rapidly acting drug. Baillieres Clin Rheumatol 4:575-593.

Uhlig T, Moe RH, and Kvien TK (2014) The burden of disease in rheumatoid arthritis. Pharmacoeconomics 32:841-851.

Address correspondence to: Dr. Leslie Chinn, Department of Clinical Pharmacology, Genentech, Inc., One DNA Way, South San Francisco, CA 94080. E-mail: chinn.leslie@gene.com 